\title{
International Journal of Clinical Therapeutics and Diagnosis (IJCTD)
}

ISSN 2332-2926

\section{Endometrial Cancer - Diagnosis}

George D. Michail

University of Patras Rion, Greece.

*Corresponding Author:

George D. Michail,

University of Patras Rion, Greece.

E-mail: gr.michail@yahoo.com

Received: January 11, 2015

Accepted: April 21, 2015

Published: April 23, 2015

Citation: George D. Michail (2015) Endometrial Cancer - Diagnosis. Int J Clin Ther Diagn. S1:005 17-27. doi: http:/ / dx.doi.org/10.19070/23322926-SI01005

Copyright: George D. Michail ${ }^{\circ}$ 2015. This is an open-access article distributed under the terms of the Creative Commons Attribution License, which permits unrestricted use, distribution and reproduction in any medium, provided the original author and source are credited.

\section{Epidemiology}

Endometrial cancer (EC) ranks as the fourth most common cancer among women and the most common malignant neoplasm of the female genital tract in the USA [1]. It most commonly occurs after the age of 50 and prognosis depends largely on the stage of the disease at the time of diagnosis. The overall lifetime risk of developing EC is approximately $2.5 \%$. The risk of EC in a population of asymptomatic postmenopausal women is reported to be $0.2 \%[2]$.

Most commonly (90\%) EC presents as postmenopausal bleeding (PMB), although only $10 \%$ of women with PMB will eventually have cancer [3]. Another common symptom is persistent postmenopausal vaginal discharge due to pyometra. Pre-menopausal women with EC usually present with significant alterations in menstrual pattern, or with an incidental finding of abnormal endometrial cells on routine cervical cytology.

Histopathological examination of hysterectomy specimens revealed that $20 \%$ to $43 \%$ of patients with a previous atypical endometrial hyperplasia $(\mathrm{AEH})$ biopsy also harbored EC $[4,5]$. Some studies consider EC and AEH as one entity, given that $40 \%$ of cases of AEH might have concurrent EC. There is limited concordance between pathologists on the diagnosis of $\mathrm{AEH}$, and the latter is considered a precancerous state with more than $25 \%$ of patients progressing to EC (estimates range from $25 \%$ to $82 \%$ ) [6-9].

Studies that aimed to evaluate the frequency of unexpected diagnose of EC made postoperatively in surgical specimens for a presumed benign condition conclude that the rate of unanticipated EC during hysterectomy performed to treat benign conditions is generally low [10].

Besides the established predisposing factors for Type $1 \mathrm{EC}$, other related epidemiologic factors are older age, higher mean BMI, late menopausal status, prolonged bleeding, and multiple episodes of PMB [11]. In Salman et al [11] study, among women with recurrent bleeding, $23.3 \%$ had a final diagnosis of EC, while only $1.4 \%$ of women with single bleeding episode had EC. Additionaly highrisk population is represented by patients with Lynch syndrome, Cowden syndrome, obesity, diabetes, or breast cancer on tamoxifen medication. On the contrary, oral contraceptive pill use, increased age at menarche, and high parity were associated with a decreased risk [8].

Some scoring systems have been developed for the prediction of EC. Angioli's et al "risk of endometrial malignancy (REM) score", [12] is capable to classify patients with endometrial abnormalities into high- or low-risk groups for EC using serum markers, ultrasound, and clinical features. Implementing this tool, the authors aimed to identify the appropriate timing of imaging and surgery in a more personalized manner. The score encompassed patient's age, sonographic endometrial thickness (ET) measurement, and blood HE4 \& CA125 levels [12].

\section{Histology \& Receptors}

Traditional classification of EC is based either on clinical and endocrine features (e.g. Types 1 and 2) or on histopathological characteristics (e.g. endometrioid, serous, or clear-cell adenocarcinoma). Subtypes defined by the different classification systems correlate to some extent, but there is substantial heterogeneity in biological, pathological, and molecular features within tumor types among various classification systems [13].

Thus, about $80 \%$ of EC's are histologically classified as Type 1 endometrioid carcinomas. These illustrate minimal myometrial invasion, arising from atypical complex hyperplasia, and especially affect pre- and perimenopausal women. Type 1 EC's are usually estrogen-receptor-positive tumors associated with hyperestrogenism and prior estrogenic stimulation [14]. In contrast, Type 2 EC's occur mostly in elderly or postmenopausal non-obese women, are more aggressive with poor prognosis, and are frequently not associated with high estrogen levels [14].

Based on the World Health Organization (WHO) categorization, the severity of endometrial hyperplasia is classified as simple or complex (based on architectural features), and as typical or atypical (based on cytologic features), as initially described by Kurman et al [9] Atypical hyperplasia (AEH) is considered a precursor lesion, which may progress to EC in $5 \%$ to $25 \%$ of patients. Several studies have reported on the coexistence of atypical hyperplasia 
with EC [14].

The benign byperplasia $(\mathrm{BH})$ and endometrial intraepithelial neoplasia (EIN) schema has also gained some acceptance among pathologist and clinicians. The research leading to the EIN criteria is based on molecular and progression data. The BH/EIN schema has better reproducibility among pathologists, is intuitively easy to use, but requires understanding of endometrial physiology and neoplastic alterations [15].

\section{Mass Population Screening}

Although many (especially Type 1) EC's develop by way of a precursor lesion (i.e. AEH), routine mass screening of the population for endometrial malignancy (most probably with pelvic ultrasound scans, biomarkers, or least likely with endometrial biopsies) is impractical and not cost-effective due to the low prevalence of the precursor disease. Thus, management relies on the prompt assessment of symptomatic women, especially those at high risk. It is imperative to promptly evaluate individuals past their fourth decade of life if there is abnormal vaginal bleeding [3].

Jacobs et al [8] reported recently on the results of a large UK multicenter prospective trial of ovarian cancer screening, one arm of which involved pelvic ultrasound during which endometrial thickness was measured, as EC screening among postmenopausal women. Despite concluding that transvaginal sonography (TVS) screening for EC has good sensitivity in postmenopausal women, they also consider that the role of population screening for EC remains currently uncertain.

Similarly, many authorities either do not recommend routine screening for EC in asymptomatic women, or consider that routine TVS or endometrial sampling do not result in lower mortality rates from EC [16].

\section{Endometrial Cancer In Premenopausal Patients}

Despite the majority of EC's affecting predominantly women of the $6^{\text {th }}$ and $7^{\text {th }}$ decade, some $20-25 \%$ of cases are diagnosed in premenopausal women [17].

When $12 \%-25 \%$ of all EC's occur in premenopausal women, only $1-5 \%$ are found in women younger than 40 years of age [18-21]. The incidence of EC has a distinct increase over the age of 35 years. Based on age alone endometrial assessment to exclude cancer is indicated in any woman older than 35 years who is suspected of having anovulatory uterine bleeding. In addition, although EC is less common under the age of 35 years, it is not absent. Thus, in high-risk patients younger than 35 years endometrial evaluation might be warranted [22].

Premenopausal woman with EC present with some pattern of abnormal uterine bleeding (AUB). Abnormal uterine bleeding is any abnormal bleeding occurring in pre- and postmenopausal women due to several causes such as organic (polyps, myomas, hyperplasia, atrophy, EC, etc) or not organic (dysfunctional uterine bleeding, luteal phase defect, etc) [23]. AUB is a broad term encompassing a range of presentations: 1) Menorrhagia, i.e. heavy cyclical bleeding over several consecutive cycles, or menstrual blood loss greater than $80 \mathrm{~mL}$ per cycle, 2) Postcoital bleeding, i.e. genital tract bleeding after intercourse 3) Intermenstrual bleeding, i.e. arising from the genital tract, not during menstruation or following intercourse in a woman having periods [24]. However, a certain percentage of women with Type 2 EC might not present with bleeding [25].

Even if there is no consensus about which imaging modality represents the most efficient way of managing post-menopausal women with abnormal bleeding, TVS, and/or SIS if needed, represents a simple, cheap and accurate first-line investigation for this group of women [20]. Studies in cycling premenopausal women found no cases with EC with ET values ranging from 4 to $8 \mathrm{~mm}$ $[22,26,27]$. Of note is that the current ACOG Committee Opinion for premenopausal women [24] considers measurement of ET in premenopausal women unhelpful in the evaluation of AUB.

For persistent AUB in women $\geq 40$ years without focal endometrial pathology in TVS an endometrial biopsy is warranted to exclude global endometrial pathologies such as endometrial hyperplasia (AEH or not) or EC [20].

\section{Endometrial Cancer In Peri \& Postmenopausal Patients}

In the Western World, EC is the most common gynecologic malignancy and 85-95\% of women harboring EC present with postmenopausal bleeding (PMB) [28, 29]. PMB represents an AUB variant defined as genital tract bleeding occurring at least 12 months after the last menstrual period of amenorrhoea. The prevalence of PMB is approximately $10 \%$ in the years immediately after menopause [28].

Older statistics estimate the risk of EC in a 50-year-old woman with $\mathrm{PMB}$ is $9 \%, 16 \%$ for a woman in her sixties, $28 \%$ for a woman in her seventies, and 60\% for a woman in her eighties [30].

For several decades the belief was that PMB "is EC until proven otherwise". However, this statement has not stood the test of time; as most statistics illustrate that the incidence of malignancy in patients with PMB ranges from $1 \%$ to $14 \%[20,22]$. The majority of patients with PMB actually bleed secondary to atrophic changes of the vagina or endometrium [31]. TVS measurements have illustrated that the average ET of a normal atrophic uterus is approximately $2.3 \mathrm{~mm}[16]$.

As a rule, acute and severe PMB bleeding is relatively uncommon [20]. Smith et al [32] who studied a cohort of women with recurrent PMB estimated that the risk of concealed EC or AEH was significantly less in women with recurrent PMB (9\%) as compared with those with a first episode of PMB (8\%), but were significantly more likely to harbor benign endometrial polyps $(28 \%)$ compared with women with a first episode of PMB $(19 \%)$.

In most algorithms, dilatation and curettage (D\&C), the longstanding principal method of endometrial investigation for several decades, has been now almost completely superseded by endometrial biopsy and hysteroscopy. TVS has been introduced to reduce the invasiveness of investigatory procedures.

Despite the numerous studies suggesting various algorithms on the investigation of PMB, there is still no consensus on the most accurate and efficient diagnostic pathway. Similarly, several scientific societies publish national and international guidelines describing different flowcharts in the diagnostic work-up of women with PMB [33-37]. Most guidelines concur that when a patient presents with $\mathrm{PMB}$ the first step in is referral to a gynecologic practice for examination, cytology and TVS. Only the US guide- 
lines $[34,35]$ "recommend either TVS or outpatient endometrial sampling as the first step in diagnosing women with PMB" [28], based on similar sensitivities and cost-effectiveness for the detection of EC for ET $\geq 5 \mathrm{~mm}$ and for endometrial sampling when 'sufficient' tissue is obtained [21, 38].

In most other current guidelines the first step is TVS, based on the high sensitivity and non-invasive character of the procedure [28].

\section{General Assessment}

In general terms, examining the patient consists of:

i) General inspection \& examination,

ii) Abdominal palpation,

iii) Speculum examination, and

iv) Bimanual pelvic examination.

\section{Assessment of the Uterine Cavity}

\section{Endometrial Sampling}

For premenopausal patients, the current ACOG Committee Opinion No 128 ("Diagnosis of Abnormal Uterine Bleeding in Reproductive-Aged Women") [24] indicates office endometrial biopsy as the first-line procedure for tissue sampling in the evaluation of AUB patients. Thus, endometrial tissue sampling should be performed in patients with AUB who are older than 45 years as a first-line test. Endometrial sampling should be also performed in patients younger than 45 years with a history of unopposed estrogen exposure, failed medical management, and persistent AUB. A positive endometrial sampling result is more accurate for ruling in disease than a negative test result is for ruling it out. Aiming to exclude EC, according to the above publication a clinician should also consider: i) To perform endometrial tissue sampling in patients with AUB who are older than 45 years as a first-line test, ii) To resort to TVS as the initial screening test for AUB and MRI as a second-line test to be used when the diagnosis is inconclusive, when further clarification would affect patient management, or when coexisting uterine myomas are suspected, and iii) To further test women with persistent bleeding with a previous benign pathology to rule out non-focal or a structural endometrial pathology (all the above are classified as Level C evidence).

Blind endometrial biopsy could either represent first line assessment [34, 35], or could follow hysteroscopy [39]. When salineinfusion hysterosonography (SIS) is used, subsequent blind endometrial biopsy is performed only for suspected $\mathrm{EC} / \mathrm{AEH}$, or imaging illustrating secretory/proliferative endometrium, or to confirm the patterns of an atrophic endometrium [39].

The pooled data of the Dijkhuizen's meta-analysis [40] (with end-point EC, and 39 studies with both pre- and postmenopausal women) illustrate that outpatient endometrial sampling is a highly sensitive technique for diagnosing endometrial carcinoma [40]. According to the meta-analysis of Dijkhuizen et al. on the diagnostic accuracy of different sampling devices, endometrial biopsy with the Pipelle device is superior to other techniques in the detection of EC and atypical hyperplasia. The detection rate for EC of Pipelle was $99.6 \%$ and $91 \%$ in post-menopausal and premenopausal women, respectively $[20,40]$.
Clark et al [41] studied the diagnostic accuracy of outpatient endometrial sampling in detecting endometrial hyperplasia. Using a variety of devices, the authors conclude that the pre-test probability for endometrial hyperplasia of $14.3 \%$ was increased to a post-test probability for a positive result of $66.7 \%$. Failure rate was $17 \%$ (unsuccessful endometrial sampling or unfeasible/not possible for technical reasons) while inadequate sampling rate (insufficient tissue obtained for a safe pathologic diagnosis) reached $7 \%$. This review demonstrated that endometrial sampling is moderately accurate in diagnosing (pre)malignant endometrial pathology. Thus, a positive test result was more accurate than a negative test result (sensitivity $91.9 \%$, with a specificity of $99.7 \%$ ).

It should be kept in mind, that when using Pipelle sampling, the proportion of total endometrial surface area sampled is about $4 \%$ (range, $0 \%-12 \%$ ) of the endometrial lining [42]. Consequently, endometrial biopsy can potentially detect most cases in which the tumor involves more than half of the endometrium, but may miss tumors involving less surface area [43]. Therefore, endometrial biopsy can reliably detect EC involving a large portion of the endometrium, but is suboptimal for evaluation of focal lesions. As a result, some discrepancy of EMB's performed in the office with the final diagnosis is anticipated and has been documented [44].

Office-based endometrial biopsy can be uncomfortable, is unsuccessful in $2-28 \%$ of attempts (secondary to cervical stenosis because of a small introitus, pain, or intolerance with the procedure) and might be inconclusive (non-diagnostic), besides it often yields an inaccurate diagnosis, particularly for women with polyps. In those situations, a D\&C procedure is required to reach a diagnosis [21].

\section{D-GRAY Scale Transvaginal Sonography}

The assessment of endometrial morphology and ET on TVS in post-menopausal women represents the cornerstone of management in current clinical practice worldwide. On ultrasonography, EC is associated with a thickened endometrium caused by the growth of tumor tissue and/or concomitant hyperplasia [20]. Cut-offs for ET of $<4 \mathrm{~mm}$ or $<5 \mathrm{~mm}$ have been prospectively validated [20].

TVS is less invasive, well tolerated, generally painless, without complications and non-diagnostic only in a small percentage of patients [21]. Usually allowing visualization of the entire endometrial cavity, TVS misses fewer abnormalities than office-based endometrial-biopsy [21]. TVS has similar sensitivity as endometrial biopsy and can be used when endometrial biopsy is unavailable, non-diagnostic or unsuccessful [21]. It is highly likely that at prevalence rates of neoplastic disease typically encountered in modern clinical practice, TVS as the initial examination yields cost savings in comparison with biopsy-based models [39].

TVS performs better at detecting EC than polyps or hyperplasia [21]. Despite being more accurate among non-HRT users [21], it is equally effective in the identification of endometrial disease regardless of HRT use [21]. A negative ET test result can reduce the posttest probability of EC to $2.4 \%(95 \%$ CI 1.3-3.9) at $\geq 4 \mathrm{~mm}$ and $5.0 \%$ (95\% CI 2.9-9.1) at $\geq 5 \mathrm{~mm}$ [16]. Furthermore, TVS is well appointed to assess persistent bleeding despite a histological diagnosis of atrophy; a low ET supports the diagnosis of atrophy, whereas a thickened measurement indicates inadequate sampling and possible existing missed lesions [21]. 
A woman with PMB has a pretest probability of $10 \%$ for EC [22, 45]. An ET $\leq 4 \mathrm{~mm}$ reduces the post-test probability to $1.2 \%$, and an $\mathrm{ET} \leq 3 \mathrm{~mm}$ reduces the post-test probability to $0.7 \%[28,45]$. To allow for reliable evaluation of the endometrium, TVS has to be performed before endometrial sampling [20].

At a $3 \mathrm{~mm}$ cut-off, ET detected $98 \%$ of women with endometrial disease, but also identified $38 \%$ of women with normal histologic findings as abnormal [21]. In postmenopausal women, an ET $\leq$ $3 \mathrm{~mm}$ has the greatest sensitivity to exclude EC, although using a cutoff of $\leq 4 \mathrm{~mm}$ might be more cost-effective [22, 28, 45].

With a threshold of $5 \mathrm{~mm}$ for ET, the sensitivity for detecting any endometrial disease was $92 \%$, and the sensitivity for detecting EC was 96\%. Similarly, in another ultrasound screening study of women without bleeding, a cutoff of $5 \mathrm{~mm}$ had a positive predictive value of $1.4 \%$, and for $10 \mathrm{~mm}$, the positive predictive value was $4.5 \%$; the negative predictive value was $99.9 \%$ for both cutoffs [8].

Based on the theoretical model created by Smith-Bindman in 2004 [46], in a postmenopausal woman with vaginal bleeding, the risk of EC is approximately $7.3 \%$ with thick $(>5 \mathrm{~mm}$ ) endometrium and $<0.07 \%$ with thin endometrium $(\leq 5 \mathrm{~mm}$ ). In postmenopausal women without vaginal bleeding, thresholds are different; the risk of EC is approximately $6.7 \%$ with thick endometrium $(>11$ $\mathrm{mm})$ and $0.002 \%$ with thin endometrium $(\leq 11 \mathrm{~mm})$. Assuming the above, in a symptomless postmenopausal woman, if the ET $\leq 11 \mathrm{~mm}$, the risk of EC is low, and endometrial biopsy is not necessary [47].

However, TVS is less appropriate and effective for evaluating intracavitary pathology (fibroids, polyps etc) [26, 31, 48]. A well-defined endometrial echo on a saggital TVS plane might be indistinguishable even in $10 \%$ of perimenopausal patients [26]. Non-diagnostic tests can occur more often in women with EC in which the endometrium is difficult to visualize [21]. Incomplete or insufficient imaging can also occur in women with previous uterine surgery (e.g., myomectomy, endometrial ablation), coexisting fibroids, morbid obesity, or an axial uterus [31, 43]. Incomplete visualization of the endometrium, an ill-defined endometrium as well as suspicion of focal lesions on scan, should be all considered abnormal. In such cases the examination should be considered inconclusive and these women need some kind of additional evaluation. If the endometrium cannot be assessed over the whole length of the uterine cavity, or the echogenicity of the endometrium is not homogeneous, Bignardi et al [20] propose to proceed with a SIS procedure.

Tabor et al [49] consider that ET measurement in symptomatic women (PMB) does not reduce the need for invasive diagnostic testing because $4 \%$ of EC's would still be missed (false-negative rate), with a false-positive rate as high as $50 \%$. Thus they do not recommend performing TVS prior to invasive testing in the setting of evaluation of PMB.

TVS can be also used to assess myometrial invasion and cervical involvement in uterine carcinoma with a greater than $90 \%$ negative predictive value [50]. Another important consideration, in addition to ET measurement, is the assessment of endometrial contour and texture. If they are heterogeneous and irregular, those parameters could be a more important determinant than absolute
ET values [31, 51].

Addressing Type 2 EC, Wang et al [52], documented that only $65 \%$ of their patients had a thickened endometrial echo-complex. Seventeen percent of patients had an endometrial echo-complex measuring $<5 \mathrm{~mm}$ and another $17 \%$ had an indistinct endometrial lining. Thus, they conclude that a thin endometrial echo complex on ultrasound does not reliably exclude Type 2 EC's.

\section{SIS (Saline Infusion Sonography - Sonohystero- gram - Sonohysterography)}

SIS is an office-based procedure providing additional information with minimal risks. The principle is of infusing saline or other distention medium into the uterine cavity immediately before performing TVS, thus enhancing the structure's contour and therefore augmenting their characterization. SIS can differentiate normal anatomic findings, globally thickened endometrium amenable to endometrial biopsy, and focal abnormalities best assessed by hysteroscopy $[43,53]$. Bignardi et al [20] point that SIS is most valuable in the detection of focal intra-cavitary lesions. Occasionally, SIS can provide sufficient information to avoid an unnecessary hysteroscopy; in practical terms SIS is used to distinguish between a diffusely thickened endometrium, for which D\&C could be the next step, and between a focal lesion, for which a hysteroscopy is the next optimal procedure.

SIS is highly sensitive (95\%) and specific (88\%) for EC [26, 53]. It is related with $7 \%$ procedure failures; some $3 \%$ of women experience pain. Vasovagal symptoms are infrequent; while a separate procedure is needed for obtaining tissue diagnosis $[22,53]$.

In an older systematic review, by De Kroon et al [54] the overall success rate of SIS was significantly lower in postmenopausal women: $87 \%$, compared with $95 \%$ for premenopausal women. The authors however conclude that SIS, in combination with an aspiration biopsy in selected cases, could represent the standard diagnostic procedure in pre- and postmenopausal women with abnormal uterine bleeding.

SIS has its limitations, however; being related with significant technical difficulties if there is significant cervical stenosis, if the uterus cannot be distended with fluid, or if the endometrium cannot be visualized otherwise $[23,26,53]$. Because of the high correlation of filling defects with EC, additional evaluation is mandatory if the uterus is unable to fill with fluid [53].

\section{Color Doppler Transvaginal Sonography}

The addition of Color Doppler to TVS aids usefully in the initial management of abnormal uterine or PMB [20]. However, Doppler ultrasonography has demonstrated rather low specificity for detection of pathologic endometrial findings, being unable to differentiate EC from endometrial polyps even when lesion size and both resistance and pulsatility indexes were considered [29].

Visualization of the pedicle artery at transvaginal color Doppler imaging (detection of a feeding blood vessel among the central endometrial echo) has a specificity of $95 \%$ for detection of endometrial polyps and a negative predictive value of 94\% [29]. An older study estimated the sensitivity of the pedicle artery sign in identifying endometrial polyps was only $76.4 \%$, which is less than the accuracy of SIS [55]. Bignardi et al [20] propose to proceed with a SIS, if the presence of a pedicle artery is suspected at color 
Doppler examination.

\section{D Volume Transvaginal Sonography}

Mansour et al [56] assessed endometrial volume as a predictor of endometrial malignancy in women with PMB. The authors acquired and calculated endometrial volume by virtual organ computer-aided analysis (VOCAL software) and concluded that an endometrial volume $\geq 1.35 \mathrm{~mL}$ might predict malignancy in women with PMB.

Yaman et al [57] conducted a study to evaluate the role of 3DTVS in diagnosing EC in PMB patients and compare its effectiveness with 2D-TVS. They consider that volume measurement by 3D-TVS has a higher specificity for this purpose, possessing improved ability to identify the negative cases compared to conventional ultrasound. They consider 3D-TVS as a helpful tool for diagnosing EC among PMB patients.

Jantarasaengaram et al [58] evaluated the accuracy of 3D-TVS with volume contrast imaging (VCI) for preoperative assessment of depth of myometrial invasion and cervical involvement in women with EC. They consider VCI-enhanced 3D-TVS as an uncomplicated method capable of predicting both parameters with reasonable accuracy in EC patients.

\section{D Power Doppler Endometrial Assessment}

Odeh and co-workers [59] assessed the accuracy of endometrial volume measurement and 3D-Power Doppler analysis (3D-PDA) in the diagnosis of EC and endometrial hyperplasia in women with peri- and PMB. They illustrated that endometrial volume acquisition and 3D-PDA are good diagnostic tools in predicting these conditions in these patient groups.

Alcazar et al [60] conducted a study to evaluate the role of 3DPower Doppler angiography (3D-PDA) to discriminate between benign and malignant endometrial disease in women with PMB and thickened endometrium. Their results show that 3D-PDA may be useful discriminator for the prediction of EC in women with PMB and increased ET at baseline sonography.

Dueholm et al [61] conducted a well-designed study to evaluate the diagnostic efficiency of 2-dimensional and 3-dimensional TVS (2D-TVS, 3D-TVS), power Doppler angiography (2D-PDA, 3DPDA), and gel infusion sonography (2D-GIS, 3D-GIS) at offline analysis for recognition of malignant endometrium compared to live-scanning; and to determine optimal image parameters at 3Danalysis. Their results indicate that live scanning is most efficient, but offline $2 \mathrm{D}$ and $3 \mathrm{D}$-analysis is useful for prediction of $\mathrm{EC}$ when optimal image quality can be obtained. They consider that the diagnostic efficiency at 3D-analysis may be improved by use of Risk of Endometrial Cancer (REC) scoring systems, without resorting to calculation of vascular indices or endometrial volume.

\section{Hysteroscopy}

Diagnostic hysteroscopy with directed biopsy has been found to be the most sensitive and specific method of diagnosing EC, other than hysterectomy $[62,63]$. Biopsy of focal lesions is performed under direct visualization. Diagnostic hysteroscopy can be performed in the office, often without sedation, whereas op- erative hysteroscopy is usually performed in the operating room under anesthesia. Clark et al [64] conducted a large meta-analysis to assess the accuracy of hysteroscopy in the diagnosis of EC and hyperplasia encompassing both pre- and postmenopausal women with AUB. A positive hysteroscopy result increased the probability of EC to $71.8 \%$, whereas a negative hysteroscopy result reduced the probability of cancer to $0.6 \%$; the authors conclude that the diagnostic accuracy of hysteroscopy is high for EC, but only moderate for any endometrial disease (EC or hyperplasia).

In the study of Ewies et al [65], the authors subjected most eligible patients to outpatient hysteroscopy $(\mathrm{OPH})$. Patients were selected for inpatient hysteroscopy (IPH) and curettage on the basis of unfeasibility of OPH (e.g. technical difficulties, woman's intolerance to pain), woman's request, inconclusive OPH results, (e.g. poor view on using $\mathrm{CO}_{2}$ hysteroscopy), or when an endometrial polyp was to be removed. The authors consider hysteroscopy is a reliable method for evaluating women with $\mathrm{PMB}$, with $90 \%$ sensitivity, $94 \%$ specificity, $92 \%$ positive predictive value, $96 \%$ negative predictive value and an overall success rate of $96.9 \%$.

The van Dongen meta-analysis [62] illustrated that diagnostic hysteroscopy is accurate in the diagnosis of intrauterine abnormalities. Other studies using hysteroscopy $[66,67]$ have indicated that endometrial polyps measuring $>15 \mathrm{~mm}$ are commonly associated with endometrial hyperplasia or EC. Other authors [44] consider hysteroscopy findings most important when ultrasound and EMB disagree.

Litta et al [23] consider outpatient hysteroscopy with biopsy as a mandatory procedure in all PMB women because of the high diagnostic accuracy with a sensibility of $100 \%$, specificity of $49.6 \%$, positive predictive value $81.3 \%$. For Schmidt et al [68] hysteroscopy represents an easy, safe and effective method in the investigation of asymptomatic women with a thickened endometrium in TVS. The commonest pathology they documented in their study was endometrial polyps.

Studies on diagnostic hysteroscopy have demonstrated low rates of serious complications and adverse events (e.g. vasovagal collapse, creation of false tracts, uterine perforation, etc) [62, 63]. As for operative hysteroscopy which is usually performed under general anesthesia, surgical risks are higher than diagnostic hysteroscopy because of resection of lesions [43]. Diagnostic hysteroscopy and SIS are accurate and safe, equally invasive, but less costeffective than endometrial biopsy [22, 62, 68]; however highly (95\%) sensitive and specific (90\%) for EC [53, 63].

However, besides a false negative rate of 3\% hysteroscopy illustrates some more shortcomings: it is invasive, expensive, while there are concerns that instrumentation spreads malignant cells into the peritoneal cavity. Some authors believe that it could be reserved to cases where an intrauterine lesion was diagnosed on TVS, or when TVS and/or EB are inconclusive. Bignardi et al [20] advocate that TVS with or without SIS can provide enough information to avoid an unnecessary hysteroscopy.

\section{Dilatation and Curettage (D\&C)}

In current clinical practice Dilatation and Curettage (D\&C) should not be used routinely for women with PMB [20]. Besides requirements for an inpatient setting, general anesthesia, operating room time and costs associated with the procedure [69] documented concerns exist over the ability of the procedure to yield a 
representative harvest of the entire endometrial cavity.

The diagnostic power of “blind" D\&C without prior hysteroscopy is limited with regard to the detection of intracavitary pathologies [70]. In a hallmark study published several decades ago, Stock \& Kanbour illustrated that in $16 \%$ of surgical specimens subjected to $\mathrm{D} \& \mathrm{C}$ less than one-quarter of the cavity has been adequately curetted, in $60 \%$ of specimens less than one-half of the cavity was curetted, and in $84 \%$ of specimens less than three quarters of the endometrial cavity was effectively curetted [70]. Tabor et al [49] indicate that endometrial malignancy may be missed by D\&C with a false negative rate ranging from $2 \%$ to $11 \%$.

The withdrawn previous National Guideline Clearinghouse stated that D\&C should be performed in women with PMB only when endometrial sampling is indicated and cannot be performed or is inconclusive and sonographic techniques are non-reassuring. In an older publication, if ET was $\geq 7.0 \mathrm{~mm}$ and the SIS procedure was negative, then formal D\&C under general anesthesia was considered superior to outpatient Pipelle endometrial sampling [53].

Thus, very seldom should $\mathrm{D} \& \mathrm{C}$ be conducted as a stand-alone procedure any longer. Schmidt et al recommend that hysteroscopy should precede D\&C to increase diagnostic efficacy in such patients. Van Hanegem et al [28] advise that D\&C should always be combined with hysteroscopy, in cases of focal pathology.

\section{Computed Tomography (CT)}

Abdominal computed tomography (CT) is not indicated as a frontline test for establishing the diagnosis of EC; indeed performing a CT scan in the context of PMB diagnostic work-up very rarely provides additional information. However severe unanticipated endometrial disease might become evident when abdominal CT scans are performed in the investigation of other, unrelated symptoms.

In contrast, CT scans are indicated for investigating the presence of cervical extrapelvic metastatic disease from EC [71].

\section{Magnetic Resonance Imaging (MRI)}

MRI has been established for use in assessing the depth of myometrial invasion in EC because of its multiplanar capacity and its excellent soft contrast resolution. A preoperative pelvic MRI contributes to accurate staging, allowing planning for the scale of surgery and preoperative counseling, especially when fertilitysparing surgery is contemplated $[72,73]$.

Preoperative MRI has been suggested as an alternative to surgical staging to guide decisions on the need for lymphadenectomy and planning the scale of surgery [74]. In Wu et al study [72], when the junctional zone in the T1 and T2-weighted images was intact, the tumor was considered noninvasive. In the dynamic films, disruption or irregularity on the subendometrial enhancement was indicative of myometrial invasion. When the signal intensity of the tumor by either T2 or dynamic study penetrated more than half of the myometrium, this was considered deep myometrial invasion from the tumor.

Hwang et al [73] reviewed several studies implementing MRI for EC preoperative evaluation and detection of deep myometrial in- vasion; they documented a wide range of sensitivity and specificity values in MRI's performance in this respect. Manfredi et al [75] illustrated that conventional MRI combined with contrastenhanced dynamic imaging showed excellent sensitivity, specificity, PPV, and NPV results.

Dynamic contrast-enhanced MRI currently probably represents the best modality to assess cervical involvement in EC $[76,77]$. Finally, when biopsy results are inconclusive regarding the true origin of a uterine neoplasm, MRI is highly specific in determining primary cervical versus EC [78].

According to Lai et al [79], MRI illustrates excellent performance in the following parameters: Early detection of EC, differential diagnosis, staging, clarification if the neoplasm is limited to endometrium or invades less than one-half or one-half or more of the myometrium, uterine serosa invasion, vaginal involvement (direct extension or metastasis), invasion to bowel mucosa, distant metastasis recognition, prognosis, response evaluation, surveillance, management of recurrence. MRI's ability to illustrate adnexal direct extension or metastasis, parametrial involvement or invasion to bladder mucosa is yet unknown.

As for cervical cancer, the intergroup study conducted by the American College of Radiology Imaging Network (ACRIN) 6651/Gynecologic Oncology Group (GOG) No 183 illustrated that, in patients with early-stage cervical cancer scheduled for curative radical hysterectomy, MRI is superior to CT by ROC analysis for evaluating uterine body involvement and measuring tumor size $[79,80]$ with sensitivity of $53 \%$ and a specificity of $74 \%$ in detection of parametrial invasion ( $\geq \mathrm{IIB})$. A European multicenter trial that aimed to evaluate tumor delineation by MRI in earlystage cervical cancer showed that the agreement between MRI and histology was good for classifying tumors and detecting deep stromal invasion, but only moderately accurate in assessing parametrial invasion [79, 81].

\section{Positron Emission Tomography (PET)}

Positron emission tomography (PET) can provide functional or metabolic information with specifically labeled radiotracers suitable for different EC disease scenarios. Integrated PET and computed tomography (PET/CT) scan using radiotracers (e.g. 18F-fluorodeoxyglucose - FDG) is now widely used clinically [79] The introduction of hybrid PET/MRI benefits from low radiation dose and high soft tissue contrast, and possesses enormous potential.

According to Lai et al [79], PET/CT illustrates excellent performance in the following parameters: Early detection of EC, distant metastasis recognition, prognosis, response evaluation, surveillance, and management of recurrence.

Based on the same article, PET/MRI illustrates excellent performance in the following parameters: Early EC detection, differential diagnosis, staging, clarification if the neoplasm is limited to endometrium or invades less than one-half or one-half or more of the myometrium, uterine serosa invasion, invasion to stromal connective tissue of the cervix, adnexal direct extension or metastasis, vaginal involvement (direct extension or metastasis), parametrial involvement, invasion to bowel mucosa, distant metastasis, prognosis, response evaluation, surveillance, and management of recurrence. 


\section{Biomarkers - Molecular Markers}

Several approaches have been reported in this emerging field. $\mathrm{Mu}-$ tations within PTEN are observed in a high percentage of Type 1 EC's, resulting in hyperactiviation of mTOR (mammalian target of rapamycin) [3, 82]. Since the documentation of correlations between vascular endothelial growth factor (VEGF) expression and prognostic factors in EC; inhibition of angiogenesis might also represent a mechanism for more targeted therapies [3].

EGFR overexpression has been demonstrated in both Type 1 and Type 2 EC, however, the initial results of some EGFR inhibitor trials have not been fully consistent $[83,84]$. ErbB receptors also possess an emerging role in EC $[85-88,107]$.

The metanalysis of Cheng et al [89] indicates a possible role for circulating YKL-40 (chitinase-3-like 1 protein (CHI3L1)) in the diagnosis of EC. Histochemical studies have suggested that expression of proliferation-related (Ki-67, CD105) and apoptosisrelated (Bcl-2, p53) proteins might predict the malignant potential of polyps [90, 91].

Tsukamoto et al [92] identified a set of endometrioid EC-associated micro-RNAs (miRNAs) in tissue and plasma samples by sequencing approach. The authors conclude that EEC-associated miRNAs in those samples could potentially discriminate endometrioid EC samples with high accuracy. Wang et al [93] illustrated that three miRNAs (miR-15b, -27a, and -233) have a possible clinical value in endometrioid EC diagnosis. The authors suggested that miR-27a and CA125 can be combined in a mathematical classifier as a potential non-invasive biomarker for detecting endometrioid EC. This classifier, including miR-27a and CA125, demonstrated a high accuracy in the diagnosis of endometrioid EC and might serve as a novel non-invasive biomarker in the future.

\section{Cytology}

Kinde et al [94] demonstrated that DNA mutational analyses of samples collected during the acquisition of common Pap smears may be capable of detecting ovarian and EC.

In a well-documented study, Norimatsu et al [95] illustrated that liquid-based preparation (LBP) of the endometrial lesions can be an important diagnostic tool for a variety of endometrial abnormalities; such as endometrial hyperplasia and EC.

Among others, a recent study by Novetsky et al [96] assessed the utility and management of vaginal cytology in the setting of follow-up after EC treatment. The authors conclude that vaginal cytology is of limited utility in detecting recurrent EC and suggest that Pap tests indicative of ASC-US/LSIL can be followed without resorting to colposcopy.

\section{Patients on Tamoxifen}

Tamoxifen (TMX) is a Selective Estrogen Receptor Modulator (SERM) used as an antagonist of the estrogen receptor in the breast tissue. On the endometrium, it possesses a weak estrogenic activity. In standard dosages, tamoxifen may be associated with endometrial proliferation, hyperplasia, polyp formation, invasive $\mathrm{EC}$, and uterine sarcoma. The increased relative risk of develop- ing EC for women on tamoxifen is 2-3 times higher than that of an age-matched population. Stage, grade, histology, and biology of tumors that develop in individuals treated with tamoxifen $(20$ $\mathrm{mg} / \mathrm{d}$ ) are no different from those that arise in the general population [97]. Following cessation of TMX use, the endometrium tends to return to normal [98].

Postmenopausal women receiving tamoxifen should be closely monitored for endometrial hyperplasia or EC symptoms. However, correlation is poor between ultrasonographic measurements of endometrial thickness and abnormal pathology in asymptomatic tamoxifen users because of tamoxifen-induced subepithelial stromal hypertrophy [99]. TMX induces stromal changes in the endometrium which thickens in a 'Swiss-cheese like pattern' caused by multiple intra-endometrial cysts of varying size [20]. Theoretically, tamoxifen effect on the endometrial lining is not observed before 2 years of use [100].

The recently reviewed ACOG Committee Opinion on Tamoxifen No 601 [101] states that the risks of endometrial proliferation, endometrial hyperplasia, EC, and uterine sarcomas, and any abnormal vaginal bleeding, bloody vaginal discharge, staining, or spotting should be investigated. An increased risk exists, both for premenopausal and postmenopausal women, of endometrial polyp formation following tamoxifen use. Evidence suggests the presence of high-risk and low-risk groups for development of atypical hyperplasias with tamoxifen treatment in postmenopausal women based on the presence or absence of benign endometrial polyps before therapy [101]. The same publication states that "Premenopausal women treated with tamoxifen have no known increased risk of uterine cancer and require no additional monitoring beyond routine gynecologic care". If atypical endometrial hyperplasia develops, appropriate gynecologic management should be instituted, and the use of tamoxifen should be reassessed The authors of the 2014 Committee Opinion state that "Unless the patient has been identified to be at high risk of EC, routine endometrial surveillance has not proved to be effective in increasing the early detection of EC in women using tamoxifen and is not recommended'.

Conclusively, in asymptomatic women using tamoxifen, screening for EC with routine TVS, endometrial biopsy, or both has not been shown to be effective although SIS might yield some additional information. To distinguish between an empty cavity with a cystic appearance of the subepithelial layer of the endometrium and true lesions inside the uterine cavity, SIS or hysteroscopy are recommended in case of a thickened endometrium at TVS in symptomatic TMX users [20].

Bignardi et al [20] suggested endometrial assessment prior to initiation of TMX treatment, and careful follow-up in all high-risk patients with baseline, initial lesions. Other authors [102] who studied asymptomatic women with breast cancer documented a high prevalence of baseline subclinical endometrial polyps, especially in obese postmenopausal patients with estrogen receptor positive breast cancer. They suggest a rationale for baseline pretamoxifen screening of some sort for the group of obese asymptomatic postmenopausal patients, especially if they are elderly and ER positive.

Metastatic Assessment For Newly Diagnosed Endometrial Cancers 
Given their non-invasiveness, imaging modalities (CT, MRI, PET/ CT) are the most popular method for detecting possible EC metastasis. The Society of Gynecologic Oncology's ("SGO”) Clinical Practice Committee 2014 recommendations [103] suggest: i) Routine preoperative assessment of EC patients with imaging tests evaluating for metastasis is not necessary (Level of evidence: $A$ ), and ii) Serum CA125 measurement may be useful in management planning of selected EC patients but cannot currently be recommended for routine clinical use (Level of evidence: $C$ ).

\section{Critical Appraisal of Diagnostic Strategies}

The standard diagnostic evaluation for EC includes pelvic ultrasonography (preferably TVS), office endometrial biopsy, or dilatation and curettage (D\&C) with or without hysteroscopy. However, no single test has been proven to yield a globally high diagnostic accuracy for all different patients harboring EC. Several different diagnostic algorithms have been proposed combining two or more available tests $[20,22,28,29,53,104]$. The exact sequence of investigation will depend upon local expertise and resources, clinical judgment and possibly patient preference.

Today, in everyday clinical practice in most countries, TVS would represent the reasonable first-line approach, with the probability of endometrial pathology strongly reduced with an ET $\leq 4$ $\mathrm{mm}$ so that endometrial sampling is not recommended below this threshold; even though some cancers will be inevitably missed $[11,16,21,49]$. For women with PMB with an ET at TVS $>4$ $\mathrm{mm}$, more invasive testing is required. The same applies when ET cannot be visualized adequately. Different guidelines use different cut-off values of ET, varying from 3 to $5 \mathrm{~mm}$. These cut-off points are mostly based on the older review by Gupta et al [16] and the work of Smith-Bindman [21, 46], as well as the Nordic literature.

An incidental finding of a thick endometrium on TVS in postmenopausal women is common (10-17\%) and, in the absence of PMB does not automatically require intervention [22]. In the pursuit of a failsafe histological diagnosis, clinicians should be also aware that EC might rarely arise in one of the two cornus of a bicornuate uterus which was yet unknown [105]. Additionally, there is some evidence that TVUS may be less sensitive for the detection of Type 2 EC's irrespective of the endometrial stripe thickness and morphology $[52,106]$.

Two factors that should be kept in mind, based on the author's opinion, are i) Several published algorithms fail to discriminate between symptomatic (PMB) and asymptomatic women; thresholds should be different for lean and obese patients, as well as women in the early and late menopause, and ii) As mentioned above, protocols are very often dictated by the availability of equipment, consumables, costs and physicians' skills or preferences. These considerations highlight the need for universal detailed algorithms, based on economic models and large meta-analyses.

More than 10 years ago, Medverd et al [39] pointed out a paramount economic principal, illustrating that the population prevalence of any neoplastic disease is the key factor that determines total cost-effectiveness between competing diagnostic algorithms. The authors illustrated that in populations with $31 \%$ or less combined prevalence of EC/atypical adenomatous hyperplasia, algorithms utilizing TVS as the initial test are the most cost minimizing. At combined endometrial carcinoma/atypical adenomatous hyperplasia prevalence ranging around $10 \%$, savings of up to $11 \%$ and $16 \%$ over pathways initiated with endometrial biopsy are predicted. However, in populations with a high incidence of neoplastic disease $(>31 \%)$, biopsy-based algorithms should become least costly.

The recent Society of Gynecologic Oncology's ("SGO”) Clinical Practice Committee 2014 recommendations [103] suggest that: i) Outpatient endometrial biopsy with the Pipelle catheter is reliable and accurate for the detection of disease in most EC cases (Level of evidence: A). ii) Hysteroscopic-guided endometrial biopsy remains the gold standard for EC diagnosis (Level of evidence: A). iii) TVS is highly sensitive and specific in predicting the presence of EC and can thus be used to triage patients for endometrial biopsy (Level of evidence: B). iv) Should symptoms persist despite negative findings from the previously cited tests, further evaluation is justified because none of these tests have $100 \%$ sensitivity (Level of evidence: $B$ ).

The largest older meta-analyses (Gupta, 2002) [16], with almost 10,000 patients, illustrated that an $\mathrm{ET} \leq 3 \mathrm{~mm}$ provides a posttest probability of $0.4 \%$ for EC; a $4-\mathrm{mm}$ threshold, $1.2 \%$; and a $5-\mathrm{mm}$ threshold, 2.3\% (Hierarchy of evidence: Class 2). The best quality evidence in this study was that for the $5 \mathrm{~mm}$ threshold. In short, this meta-analysis concludes that despite TVS-measured ET has limited diagnostic ability in predicting endometrial hyperplasia or EC, however it still excludes effectively most of endometrial pathology. The authors stress the need for well-appointed prospective randomized controlled trials on TVS-measured ET.

Almost ten years later, Jacobs et al [8] published on a nested-casecontrol study with prolonged follow-up of 37,000 postmenopausal participants who underwent TVS. With an ET cutoff of 5 $\mathrm{mm}$, sensitivity was $80.5 \%$ and specificity was $85.7 \%$ for EC or AEH. An increased cutoff of $10 \mathrm{~mm}$ or greater resulted in a reduced sensitivity and increased specificity

In this study [8], the logistic regression model identified 25\% of the population as at high-risk; indeed $39.5 \%$ of $\mathrm{EC}$ or $\mathrm{AEH}$ cases were actually within this specipic group. In this high-risk population, a cutoff at $6.75 \mathrm{~mm}$ achieved sensitivity of $84.3 \%$ and specificity of $89.9 \%$ for EC. In this study population, logistic regression showed that decreased $\mathrm{EC}$ or $\mathrm{AEH}$ risk was associated with the use of the oral contraceptive pill, age at menarche, and pregnancies longer than 6 months, while increased risk was associated with rising weight, increasing age, and personal history of breast and other cancer. While the authors comment that in clinical practice asymptomatic women are not investigated, the also conclude that TVS screening for EC has good sensitivity in postmenopausal women. The authors point that the burden of diagnostic procedures and false-positive results can be reduced by limiting screening to a higher-risk group; however they reckon that the role of population screening for EC remains uncertain.

\section{References}

[1]. Jemal A, Murray T, Ward E, Samuels A, Tiwari RC, et al. (2005) Cancer statistics,. CA Cancer J Clin 55(1): 10-30.

[2]. Gull B, Karlsson B, Milsom I, Wikland M, Granberg S (1996) Transvaginal sonography of the endometrium in a representative sample of postmenopausal women. Ultrasound Obstet Gynecol 7(5): 322-7.

[3]. May J, Mehasseb MK (2013) Endometrial cancer. Obstet Gynecol Reprod Med 23(9): 263-9.

[4]. DiSaia P.J, Creasman W.T (1997) Endometrial hyperplasia/estrogen thera- 
py. Clinical Gynecologic Oncology. Baltimore: Mosby-Year Book, Inc..

[5]. Creasman, W. T. (1997). Endometrial cancer: incidence, prognostic factors, diagnosis, and treatment. In Seminars in oncology. 24(1):140-46.

[6]. Wentz WB (1974) Progestin therapy in endometrial hyperplasia. Gynecol Oncol 2: 362-67.

[7]. Lacey JV Jr, Sherman ME, Rush BB (2010) Absolute risk of endometrial carcinoma during 20-year follow-up among women with endometrial hyperplasia J Clin Oncol 28: 788-92.

[8]. Jacobs I, Gentry-Maharaj A, Burnell M, Manchanda R, Singh N, et al. (2011) Sensitivity of transvaginal ultrasound screening for endometrial cancer in postmenopausal women: a case-control study within the UKCTOCS cohort. The lancet oncology 12(1):38-48

[9]. Kurman RJ, Kaminski PF, Norris HJ (1985) The behavior of endometrial hyperplasia. A long-term study of "untreated" hyperplasia in 170 patients. Cancer 56(2): 403-412.

[10]. Ouldamer L, Rossard L, Arbion F, Marret H, Body G (2014) Risk of Incidental Finding of Endometrial Cancer at the Time of Hysterectomy for Benign Condition. J Minim Invas Gynecol 21(1): 131-5

[11]. Salman MC, Bozdag G, Dogan S, Yuce K (2013) Role of postmenopausal bleeding pattern and women's age in the prediction of endometrial cancer. Aust N Z J Obstet Gynaecol 53(5): 484-8.

[12]. Angioli R, Capriglione S, Aloisi A, Luvero D, Cafa EV et al. (2013) REM (risk of endometrial malignancy): a proposal for a new scoring system to evaluate risk of endometrial malignancy. Clin Cancer Res 19(20): 5733-9.

[13]. Murali R, Soslow RA, Weigelt B (2014) Classification of endometrial carcinoma: more than two types. Lancet Oncol 15(7): 268-78.

[14]. Pessoa JN, Freitas AC, Guimaraes RA, Lima J, Dos Reis HL et al. (2014) Endometrial Assessment: When is it Necessary J Clin Med Res 6(1): 21-5.

[15]. Owings RA, Quick CM (2014) Endometrial Intraepithelial Neoplasia. Arch Pathol Lab Med 138: 484-491.

[16]. Gupta JK, Chien PF, Voit D, Clark TJ, Khan KS (2002) Ultrasonographic endometrial thickness for diagnosing endometrial pathology in women with postmenopausal bleeding: a meta-analysis. Acta Obstet Gynecol Scand 81(9): 799-816.

[17]. National Cancer Institute of Canada: Canadian Cancer Statistics 2012. (2012). Toronto, Canada.

[18]. Pellerin GP, Finan MA (2005) Endometrial cancer in women 45 years of age or younger: a clinicopathological analysis. Am J Obstet Gynecol 193(5): $1640-4$

[19]. Osmers RGW, Kuhn W (1994) Endometrial cancer screening. Curr Opin Obstet Gynecol 6: 75-9.

[20]. Bignardi T, Van den Bosch T, Condous G (2009) Abnormal uterine and post-menopausal bleeding in the acute gynaecology unit. Best Pract Res Clin Obstet Gynaecol 23(5): 595-607.

[21]. Smith-Bindman R, Kerlikowske K, Feldstein V. A, Subak L, Scheidler J, et al. (1998) Endovaginal ultrasound to exclude endometrial cancer and other endometrial abnormalities. Jama 280(17):1510-1517.

[22]. Goldstein SR (2010) Modern evaluation of the endometrium. Obstet Gynecol 116(1): 168-176

[23]. Litta P, Merlin F, Carlo C, Pozzan C, Sacco G, et al. (2005) Role of hysteroscopy with endometrial biopsy to rule out endometrial cancer in postmenopausal women with abnormal uterine bleeding. Maturitas 50: 117-123.

[24]. American College of Obstetricians and Gynecologists - ACOG COMMITTEE OPINION No 128 Diagnosis of Abnormal Uterine Bleeding in Reproductive-Aged Women (2012). Obstet Gynecol 120(1): 197-206.

[25]. Chandavarkar U, Kuperman JM, Muderspach LI, Opper N, Felix JC, et al. (2013) Endometrial echo complex thickness in postmenopausal endometrial cancer. Gynecol Oncol 131(1): 109-12.

[26]. Goldstein SR (1994) Use of ultrasonohysterography for triage of perimenopausal patients with unexplained uterine bleeding. Am J Obstet Gynecol 170(2): 565-570

[27]. Özdemir S, Çelik Ç, Gezginç K, Kıreşi D, Esen H (2010) Evaluation of endometrial thickness with transvaginal ultrasonography and histopathology in premenopausal women with abnormal vaginal bleeding. Arch Gynecol Obstet 282(4): 395-9.

[28]. Van Hanegem N, Breijer M. C, Khan K. S, Clark T. J, Burger M. P. M, et al. (2011). Diagnostic evaluation of the endometrium in postmenopausal bleeding: an evidence-based approach.Maturitas 68(2):155-164.

[29]. Spadoto Dias D, Bueloni-Dias FN, Dias R, Nahas-Neto J, Petri Nahas EA, et al. (2014) Custodio Domingues MA, Solange Peron Bueno Angela SP, Padovani CR. Usefulness of Clinical, Ultrasonographic, Hysteroscopic, and Immunohistochemical Parameters in Differentiating Endometrial Polyps From Endometrial Cancer. J Minim Invas Gynecol 21(2): 296-302.

[30]. Anderson B (1986) Diagnosis of endometrial cancer. Clin Obstet Gynecol 13: $739-50$

[31]. Goldstein SR (2009) The role of transvaginal ultrasound or endometrial biopsy in the evaluation of the menopausal endometrium. Am J Obstet Gynecol 201(1): 5-11.

[32]. Smith PP, O'Connor S, Gupta J, Clark TJ (2014) Recurrent Postmenopau- sal Bleeding: a Prospective Cohort Study.J Minim Invasive Gynecol 21(5): 799-803.

[33]. Dutch Society of Obstetrics and Gynecology (NVOG). Diagnostics in abnormal vaginal blood loss in the postmenopausal period; 2003.

[34]. Goldstein RB, Bree RL, Benson CB, Benacerraf BR, Bloss JD, et al. (2001) Evaluation of the woman with postmenopausal bleeding: Society of Radiologists in Ultrasound-sponsored consensus conference statement. J Ultrasound Med 10: 1025-36.

[35]. WITHDRAWN-American College of Obstetricians and Gynecologists ACOG Committee Opinion No. 426: the role of transvaginal ultrasonography in the evaluation of PMB (2009). Obstet Gynecol 113(2 Pt 1): 462-4.

[36]. Epstein E (2004) Management of postmenopausal bleeding in Sweden: a need for increased use of hydrosonography and hysteroscopy. Acta obstetricia et gynecologica Scandinavica 83(1):89-95

[37]. Scottish Intercollegiate Guidelines Network. Investigation of postmenopausal bleeding; 2002. http://www.sign.ac.uk/guidelines/fulltext/61/

[38]. Weber AM, Belinson JL, Bradley LD, Piedmonte MR (1997) Vaginal ultrasonography versus endometrial biopsy in women with postmenopausal bleeding. Am J Obstet Gynecol 177(4): 924-9.

[39]. Medverd JR, Dubinsky TJ (2002) Cost analysis model: US versus endometrial biopsy in evaluation of peri- and postmenopausal abnormal vaginal bleeding. Radiology 222(3): 619-27.

[40]. Dijkhuizen FP, Mol BW, Brolmann HA, Heintz AP (2000) The accuracy of endometrial sampling in the diagnosis of patients with endometrial carcinoma and hyperplasia: a meta-analysis. Cancer 89(8): 1765-72.

[41]. Clark TJ, Mann CH, Shah N, Khan KS, Song F, et al. (2001), Accuracy of outpatient endometrial biopsy in the diagnosis of endometrial hyperplasia. Acta Obstet Gynecol Scand 80(9): 784-93.

[42]. Rodriguez GC, Yaqub N, King ME (1993) A comparison of the Pipelle device and the Vabra aspirator as measured by endometrial denudation in hysterectomy specimens: the Pipelle device samples significantly less of the endometrial surface than the Vabra aspirator. Am J Obstet Gynecol 168(1 Pt 1): 55-9.

[43]. Apgar BS, Kaufman AJ, Bettcher C, Parker-Featherstone E (2013) Gynecologic Procedures: Colposcopy, Treatment of Cervical Intraepithelial Neoplasia, and Endometrial Assessment Am Fam Physician 87(12): 836-43.

[44]. Famuyide AO, Breitkopf DM, Hopkins MR, Laughlin-Tommaso SK (2014) Asymptomatic Thickened Endometrium in Postmenopausal Women: Malignancy Risk. J Minim Invasive Gynecol 21(5): 782-6.

[45]. Timmermans A, Opmeer B. C, Khan K. S, Bachmann L. M, Epstein, E, et al. (2010) Endometrial thickness measurement for detecting endometrial cancer in women with postmenopausal bleeding: a systematic review and meta-analysis. Obstetrics \& Gynecology 116(1):160-167.

[46]. Smith-Bindman R, Weiss E, Feldstein V (2004) How thick is too thick? When endometrial thickness should prompt biopsy in postmenopausal women without vaginal bleeding. Ultrasound Obstet Gynecol 24: 558-565.

[47]. Saatli B, Yildirim N, Olgan S, Koyuncuoglu M, Emekci O et al. (2014) The role of endometrial thickness for detecting endometrial pathologies in asymptomatic postmenopausal women. Aust N Z J Obstet Gynaecol 54(1): $36-40$

[48]. Skaznik-Wikiel ME, Jelovsek JE, Andrews B, Bradley LD (2010) Accuracy of endometrial thickness in detecting benign endometrial pathology in postmenopausal women. Menopause 17(1): 104-108.

[49]. Tabor A, Watt HC, Wald NJ (2002) Endometrial thickness as a test for endometrial cancer in women with postmenopausal vaginal bleeding. Obstet Gynecol 99: 663-70.

[50]. Gabrielli S, Marabini A, Bevini M, Linsalata I, Falco P, et al. (1996) Transvaginal sonography vs. hysteroscopy in the preoperative staging of endometrial carcinoma. Ultrasound in Obstetrics \& Gynecology 7(6):443-446.

[51]. G Michail, A Karahaliou, S Skiadopoulos, C Kalogeropoulou, G Terzis et al. (2007) Texture analysis of perimenopausal and post-menopausal endometrial tissue in grayscale transvaginal ultrasonography. $\mathrm{Br} \mathrm{J}$ Radiol 80(956):609-16.

[52]. Wang J, Wieslander C, Hansen G, Cass I, Vasilev I, et al. (2006) Thin endometrial echo complex on ultrasound does not reliably exclude type 2 endometrial cancers. Gynecol Oncol 101(1): 120-125.

[53]. Epstein E, Ramirez A, Skoog L, Valentin, L (2001) Transvaginal sonography, saline contrast sonohysterography and hysteroscopy for the investigation of women with postmenopausal bleeding and endometrium $>5 \mathrm{~mm}$. Ultrasound in obstetrics \& gynecology 18(2):157-162.

[54]. De Kroon CD, de Bock GH, Dieben SW, Jansen FW (2003) Saline contrast hysterosonography in abnormal uterine bleeding: a systematic review and meta-analysis. BJOG 110(10):938-47.

[55]. Timmerman D, Verguts J, Konstantinovic M. L, Moerman P, Van Schoubroeck, D, et al. (2003) The pedicle artery sign based on sonography with color Doppler imaging can replace second-stage tests in women with abnormal vaginal bleeding. Ultrasound in obstetrics \& gynecology 22(2):166171.

[56]. Mansour GM, El-Lamie IK, El-Kady MA, El-Mekkawi SF, Laban M, et 
al. (2007) Endometrial volume as predictor of malignancy in women with postmenopausal bleeding. Int J Gynaecol Obstet 99(3):206-10.

[57]. Yaman C, Habelsberger A, Tews G, Pölz W, Thomas Ebner T (2008) The role of three-dimensional volume measurement in diagnosing endometrial cancer in patients with postmenopausal bleeding Gynecol Oncol 110: 390-395.

[58]. Jantarasaengaram S, Praditphol N, Tansathit T, Vipupinyo C, Vairojanavong K (2014) Three-dimensional ultrasound with volume contrast imaging for preoperative assessment of myometrial invasion and cervical involvement in women with endometrial cancer Ultrasound Obstet Gynecol 43: 569-574.

[59]. Odeh M, Vainerovsky I, Grinin V, Kais M, Ophir E, et al. (2007) Threedimensional endometrial volume and 3-dimensional power Doppler analysis in predicting endometrial carcinoma and hyperplasia. Gynecol Oncol 106 (2):348-353.

[60]. Alcazar J. L, Galvan R (2009) Three-dimensional power Doppler ultrasound scanning for the prediction of endometrial cancer in women with postmenopausal bleeding and thickened endometrium. American journal of obstetrics and gynecology 200(1):44-e1.

[61]. Dueholm M., Holm JW, Rydbjerg S, Hansen ES, Ørtoft G. Two-dimensional and 3-dimensional transvaginal ultrasound with addition of power Doppler angiography and gel infusion sonography for diagnosis of endometrial malignancy. Ultrasound Obstet Gynecol 2014 May 26. doi: 10.1002/ uog.13421. [Epub ahead of print]

[62]. van Dongen H, de Kroon CD, Jacobi CE, Trimbos JB, Jansen FW (2007) Diagnostic hysteroscopy in abnormal uterine bleeding: a systematic review and meta-analysis. BJOG 114(6):664-675.

[63]. Garuti G, Mirra M, Luerti M (2006) Hysteroscopic view in atypical endometrial hyperplasias: A correlation with pathologic findings on hysterectomy specimens. J Minim Invasive Gynecol 13(4):325-330.

[64]. Clark TJ, Voit D, Gupta JK, Hyde C, Song F, et al. (2002) Accuracy of hysteroscopy in the diagnosis of endometrial cancer and hyperplasia: a systematic quantitative review. JAMA 288:1610-21.

[65]. Ewies AA, Musonda P (2010) Managing postmenopausal bleeding revisited: what is the best first line investigation and who should be seen within 2 weeks? A cross-sectional study of 326 women. Eur J Obstet Gynecol Reprod Biol 153: 67-71.

[66]. Lasmar BP, Lasmar RB (2013) Endometrial polyp size and polyp hyperplasia. Int J Gynecol Obstet 123:236-239.

[67]. Cavkaytar S, Kokanali MK, Ceran U, Topcu HO, Sirvan L, et al. (2014) Roles of sonography and hysteroscopy in the detection of premalignant and malignant polyps in women presenting with postmenopausal bleeding and thickened endometrium. Asian Pac J Cancer Prev 15(13): 5355-5358.

[68]. Schmidt T, Breidenbach M, Nawroth F, Mallmann P, Beyer I. M, et al. (2009) Hysteroscopy for asymptomatic postmenopausal women with sonographically thickened endometrium.Maturitas 62(2):176-178.

[69]. Penny GC. The investigation of menorrhagia. In: Disorders of the Menstrual Cycle. London: RCOG press, 2000. Chapter 16.

[70]. Stock RJ, Kanbour A (1975) Prehysteroctomy curettage. Obstet Gynecol 45:537-41.

[71]. Colombo N, Preti E, Landoni F, Carinelli S, Colombo A, et al. (2013) on behalf of the ESMO Guidelines Working Group. Endometrial cancer: ESMO Clinical Practice Guidelines for diagnosis, treatment and follow-up. Annals of Oncology 24 (Supplement 6): 633-638,

[72]. Wu WJ, Yu MS, Su HY, Lin KS, Lu KL, et al. (2013) The accuracy of magnetic resonance imaging for preoperative deep myometrium assessment in endometrial cancer. Taiwan J Obstet Gynecol 52:210-4.

[73]. Hwang JH, Lee NW, Lee KW, Lee JK (2009) Magnetic resonance imaging for assessment of deep endometrial invasion for patients with endometrial carcinoma. Aust N Z J Obstet Gynaecol 49: 537-41.

[74]. Harry VN, Deans H, Ramage E, Parkin DE, Gilbert FJ (2009) Magnetic Resonance Imaging in Gynecological Oncology. Int J Gynecol Cancer 19: 186-93.

[75]. Manfredi R, Mirk P, Maresca G (2004) Local-regional staging of endometrial carcinoma: role of MR imaging in surgical planning. Radiology 231(2):372-8

[76]. Sala E, Rockall A, Kubik-Huch RA (2011) Advances in magnetic resonance imaging of endometrial cancer. Eur Radiol 21: 468-473.

[77]. Savelli L, Ceccarini M, Ludovisi M (2008) Preoperative local staging of endometrial cancer: transvaginal sonography versus magnetic resonance imaging. Ultrasound Obstet Gynecol 31: 560-568.

[78]. He H, Bhosale P, Wei W, Ramalingam P, Iyer R (2013) MRI is highly specific in determining primary cervical versus endometrial cancer when biopsy results are inconclusive Clin Radiol 68:1107-1113.

[79]. Lai GH, Lin G, Yen TC, Liu FY (2014) Molecular imaging in the management of gynecologic malignancies Gynecol Oncol 135 (1):156-162.

[80]. Mitchell DG, Snyder B, Coakley F, Reinhold C, Thomas G, et al. (2006)
Early invasive cervical cancer: tumor delineation by magnetic resonance imaging, computed tomography, and clinical examination, verified by pathologic results, in the ACRIN 6651/GOG 183 Intergroup Study. J Clin Oncol 24(36):5687-94.

[81]. Epstein E, Testa A, Gaurilcikas A, Di Legge A, Ameye L, et al. (2013) Early stage cervical cancer: tumor delineation by magnetic resonance imaging and ultrasound - a European multicenter trial. Gynecol Oncol 128(3):449-53.

[82]. Samulak D, Grosman-Dziewiszek P, Michalska MM, Mojs E, Samulak K, et al. (2014) Evaluation of expression of the PTEN gene, oestrogen and progesterone receptors as diagnostic and predictive factors in endometrial cancer. Pathol Oncol Res 20(1):191-6.

[83]. Adonakis G, Androutsopoulos G, Koumoundourou D, Liava A, Ravazoula P, et al. (2008) Expression of the epidermal growth factor system in endometrial cancer. Eur J Gynaecol Oncol 29(5):450-454.

[84]. Androutsopoulos G, Adonakis G, Gkermpesi M, Gkogkos P, Ravazoula P, et al. (2006) Expression of the epidermal growth factor system in endometrial cancer after adjuvant tamoxifen treatment for breast cancer. Eur J Gynaecol Oncol 27(5):490-494.

[85]. Androutsopoulos G, Michail G, Adonakis G, Decavalas G (2014) ErbB receptors and ErbB targeted therapies in endometrial cancer. J Cancer Ther 5(6):483-492.

[86]. Adonakis G, Androutsopoulos G (2012) The role of ErbB receptors in endometrial cancer. Cancer of the Uterine Endometrium-Advances and Controversies, InTech 23-38.

[87]. Androutsopoulos G, Adonakis G, Decavalas G (2015) ErbB targeted therapy in endometrial cancer, Int J Clin Ther Diagn 1(2): 5-7.

[88]. Androutsopoulos G, Adonakis G, Liava A, Ravazoula P, Decavalas G (2013) Expression and potential role of ErbB receptors in type II endometrial cancer. Eur J Obstet Gynecol Reprod Biol 168(2):204-208.

[89]. Cheng D, Sun Y, He H (2014) Diagnostic role of circulating YKL-40 in endometrial carcinoma patients: a meta-analysis of seven related studies. Med Oncol 31(12):326.

[90]. Sington JD, Manek S (2002) Cytological atypia in endometrial polyps and immunostaining for p16, p53 and Ki67. Histopathology 41:86-88.

[91]. Maia H Jr, Maltez A, Studart E, Athayde C, Coutinho EM (2004) Ki-67, $\mathrm{Bcl}-2$ and $\mathrm{p} 53$ expression in endometrial polyps and in the normal endometrium during the menstrual cycle. BJOG 111:1242-7.

[92]. Tsukamoto O, Miura K, Mishima H, Abe S, Kaneuchi M, et al. (2014) Identification of endometrioid endometrial carcinoma-associated microRNAs in tissue and plasma. Gynecol Oncol 132(3):715-721.

[93]. Wang L, Chen YJ, Xu K, Xu H, Shen XZ, et al. (2014) Circulating microRNAs as a Fingerprint for Endometrial Endometrioid Adenocarcinoma. PLoS One 9(10): e110767.

[94]. Kinde I, Bettegowda C, Wang Y, Wu J, Agrawal N, et al. (2013) Evaluation of DNA from the Papanicolaou test to detect ovarian and endometrial cancers. Science Transl Med 5(167):167ra4.

[95]. Norimatsu Y, Yanoh K, Kobayashi TK (2013) The Role of Liquid-Based Preparation in the Evaluation of Endometrial Cytology Acta Cytologica 57:423-435.

[96]. Novetsky AP, Kuroki LM, Massad LS, Hagemann AR, Thaker PH, et al. (2013) The utility and management of vaginal cytology after treatment for endometrial cancer. Obstet Gynecol 121(1): 129-35.

[97]. Ravazoula P, Androutsopoulos G, Zyli P, Michail G, Kardamakis D, et al. (2006) A clinicopathologic study on patients with endometrial cancer after adjuvant tamoxifen treatment for breast cancer: a single center experience. Breast J 12(6):578-584

[98]. Senkus-Konefka E, Konefka T, Jassem J. (2004) The effects of tamoxifen on the female genital tract. Cancer Treat Rev 30:291-301.

[99]. Achiron R, Lipitz S, Sivan E, Goldenberg M, Horovitz A, et al. (1995) Changes mimicking endometrial neoplasia in postmenopausal, tamoxifentreated women with breast cancer: a transvaginal Doppler study. Ultrasound Obstet Gynecol 6:116-20

[100].Suh-Burgmann EJ, Goodman A (1999) Surveillance for endometrial cancer in women receiving tamoxifen. Ann Intern Med 131: 127 - 135.

[101]. American College of Obstetricians and Gynecologists - ACOG COMMITTEE OPINION No 601 Tamoxifen and Uterine cancer (2014). Obstet Gynecol 123(6):1394-1397.

[102].Lubián López DM, Orihuela López F, García-Berbel Molina L, Boza Novo P, Pozuelo Solís E, et al, (2014) Comino Delgado R. Endometrial polyps in obese asymptomatic pre and postmenopausal patients with breast cancer: Is screening necessary? Gynecol Oncol 133: 56-62.

[103]. Burke WM, Orr J, Leitao M, Salom E, Gehrig P, et al. (2014) SGO. Endometrial cancer: A review and current management strategies: Part I. Gynecol Oncol 134:385-392

[104]. Bachmann LM, Ter Riet G, Clark TJ, Gupta JK, Khan KS (2003) Probability analysis for diagnosis of endometrial hyperplasia and cancer in post- 
menopausal bleeding: an approach for a rational diagnostic workup. Acta Obstet Gynecol Scand 82: 564-8.

[105]. Munkhdelger J, Mia-Jan K, Cha DS, Eom M (2014) Endometrial carcinoma arising in a bicornuate uterus. Obstet Gynecol Sci 57(5):401-404.

[106]. Munro MG (2014) The Southern California Permanente Medical Group's Abnormal Uterine Bleeding Working Group. Investigation of Women with Postmenopausal Uterine Bleeding: Clinical Practice Recommendations Clinical Practice Recommendations. Perm J 18(1):55-70.

[107]. Androutsopoulos G, Adonakis G, Decavalas G (2014) Endometrial cancer: current epidemiology, detection and management, Nova Science Publishers 2014 [In Press]. ISBN 978-1-63463-163-1.

\section{Fyrther Suggested Reading (Supplementary) \\ Special Issue on \\ "Endometrial Cancer: Pathogenesis, Diagnosis and Treatment"}

Theme Edited by:

- Georgios Androutsopoulos, University of Patras, Greece androutsopoulosgeorgios@hotmail.com

- Georgios Adonakis, University of Patras, Greece adonakis@upatras.gr

- Georgios Decavalas, University of Patras, Rion, Greece gdecavalas@med.upatras.gr 